\title{
COVID-19 severity and mortality in patients with CLL: an update of the international ERIC and Campus CLL study
}

\author{
Thomas Chatzikonstantinou (1D ${ }^{1,2,103}$, Anargyros Kapetanakis ${ }^{2,103}$, Lydia Scarfó ${ }^{3,103}$, Georgios Karakatsoulis ${ }^{2,4}$, David Allsup (D) \\ Alejandro Alonso Cabrero ${ }^{6,7}$, Martin Andres (D) $^{8}$, Darko Antic ${ }^{9,10}$, Mónica Baile ${ }^{11}$, Panagiotis Baliakas ${ }^{12,13}$, Dominique Bron ${ }^{14}$, \\ Antonella Capasso ${ }^{15}$, Sofia Chatzileontiadou ${ }^{16}$, Raul Cordoba ${ }^{17}$, Juan-Gonzalo Correa ${ }^{18}$, Carolina Cuéllar-García ${ }^{19}$, Lorenzo De Paoli ${ }^{20}$, \\ Maria Rosaria De Paolis ${ }^{21}$, Giovanni Del Poeta ${ }^{22}$, Christos Demosthenous ${ }^{1}$, Maria Dimou (iD ${ }^{23}$, David Donaldson ${ }^{24}$, Michael Doubek (D) ${ }^{25}$, \\ Maria Efstathopoulou ${ }^{26}$, Barbara Eichhorst ${ }^{27}$, Shaimaa El-Ashwah ${ }^{28}$, Alicia Enrico (iD ${ }^{29}$, Blanca Espinet (D) ${ }^{30}$, Lucia Farina ${ }^{31}$, \\ Angela Ferrari ${ }^{32}$, Myriam Foglietta ${ }^{33}$, Henrik Frederiksen DD $^{34}$, Moritz Fürstenau (iD) ${ }^{27}$, José A. García-Marco (D) ${ }^{35}$, \\ Rocío García-Serra (D ${ }^{36,37}$, Massimo Gentile ${ }^{38}$, Eva Gimeno ${ }^{39}$, Andreas Glenthøj ${ }^{40}$, Maria Gomes da Silva ${ }^{41}$, Odit Gutwein ${ }^{42,43}$ \\ Yervand K. Hakobyan (D) ${ }^{44}$, Yair Herishanu ${ }^{45}$, José Ángel Hernández-Rivas ${ }_{(D)}^{46}$, Tobias Herold (iD ${ }^{47}$, Idanna Innocenti ${ }^{48}$, Gilad Itchaki ${ }^{49}$, \\ Ozren Jaksic ${ }^{50}$, Ann Janssens ${ }^{51}$, Olga B. Kalashnikova (D) ${ }^{52}$, Elżbieta Kalicińska ${ }^{53}$, Linda Katharina Karlsson (iD ${ }^{40}$, Arnon P. Kater ${ }^{54}$, \\ Sabina Kersting ${ }^{55}$, Jorge Labrador (iD ${ }^{56}$, Deepesh Lad (iD ${ }^{57}$, Luca Laurenti ${ }^{48,58}$, Mark-David Levin (D) ${ }^{59}$, Enrico Lista ${ }^{60}$, \\ Alberto Lopez-Garcia (iD ${ }^{17}$, Lara Malerba ${ }^{61}$, Roberto Marasca (D) ${ }^{62}$, Monia Marchetti (D) ${ }^{63}$, Juan Marquet ${ }^{64}$, Mattias Mattsson ${ }^{12,65}$ \\ Francesca R. Mauro ${ }^{66}$, Ivana Milosevic ${ }^{67}$, Fatima Mirás ${ }^{68}$, Marta Morawska ${ }^{69,70}$, Marina Motta ${ }^{71}$, Talha Munir ${ }^{72}$, Roberta Murru ${ }^{73}$, \\ Carsten U. Niemann (iD ${ }^{40}$, Raquel Nunes Rodrigues ${ }^{41}$, Jacopo Olivieri ${ }^{74}$, Lorella Orsucci ${ }^{75}$, Maria Papaioannou ${ }^{16}$, \\ Miguel Arturo Pavlovsky ${ }^{76}$, Inga Piskunova ${ }^{77}$, Viola Maria Popov (iD) ${ }^{78}$, Francesca Maria Quaglia (iD) ${ }^{79}$, Giulia Quaresmini (iD ${ }^{80}$, \\ Kristian Qvist ${ }^{81}$, Gianluigi Reda (DD ${ }^{82}$, Gian Matteo Rigolin (DD $^{83}$, Rosa Ruchlemer ${ }^{84}$, Gevorg Saghumyan ${ }^{44}$, Amit Shrestha ${ }^{85}$, \\ Martin Šimkovič ${ }^{86}$, Martin Špaček ${ }^{87}$, Paolo Sportoletti ${ }^{88}$, Oana Stanca ${ }^{89}$, Niki Stavroyianni ${ }^{1}$, Tamar Tadmor (iD ${ }^{90}$, Doreen Te Raa ${ }^{91}$, \\ Sanne H. Tonino ${ }^{92}$, Livio Trentin ${ }^{93}{ }^{93}$, Ellen Van Der Spek ${ }^{94}$, Michel van Gelder ${ }^{95}$, Roel van Kampen ${ }^{96}$, Marzia Varettoni ${ }^{97}$, \\ Andrea Visentin ${ }^{93}$, Candida Vitale ${ }^{98}{ }^{98}$, Ewa Wasik-Szczepanek ${ }^{99}$, Tomasz Wróbel ${ }^{53}$, Lucrecia Yáñez San Segundo ${ }^{100}$, \\ Mohamed Yassin ${ }^{101}$, Marta Coscia ${ }^{98}$, Alessandro Rambaldi ${ }^{80,102}$, Emili Montserrat ${ }^{18}$, Robin Foà (iD ${ }^{66}$, Antonio Cuneo ${ }^{83}$, \\ Kostas Stamatopoulos $\mathbb{I D}^{2,104 \bowtie}$ and Paolo Ghia $\mathbb{D}^{3,104 \bowtie}$
}

(c) The Author(s) 2021

Patients with chronic lymphocytic leukemia (CLL) may be more susceptible to Coronavirus disease 2019 (COVID-19) due to age, disease, and treatment-related immunosuppression. We aimed to assess risk factors of outcome and elucidate the impact of CLLdirected treatments on the course of COVID-19. We conducted a retrospective, international study, collectively including 941 patients with CLL and confirmed COVID-19. Data from the beginning of the pandemic until March 16, 2021, were collected from 91 centers. The risk factors of case fatality rate (CFR), disease severity, and overall survival (OS) were investigated. OS analysis was restricted to patients with severe COVID-19 (definition: hospitalization with need of oxygen or admission into an intensive care unit). CFR in patients with severe COVID-19 was 38.4\%. OS was inferior for patients in all treatment categories compared to untreated $(p<0.001)$. Untreated patients had a lower risk of death $(\mathrm{HR}=0.54,95 \% \mathrm{Cl}: 0.41-0.72)$. The risk of death was higher for older patients and those suffering from cardiac failure ( $\mathrm{HR}=1.03,95 \% \mathrm{Cl}: 1.02-1.04 ; \mathrm{HR}=1.79,95 \% \mathrm{Cl}: 1.04-3.07$, respectively). Age, CLL-directed treatment, and cardiac failure were significant risk factors of OS. Untreated patients had a better chance of survival than those on treatment or recently treated.

Leukemia (2021) 35:3444-3454; https://doi.org/10.1038/s41375-021-01450-8

\section{INTRODUCTION}

The Coronavirus disease 2019 (COVID-19) has cost the lives of more than 3 million people worldwide, since the first cases were reported in December 2019 in Wuhan, China [1]. While the severity of the disease can vary greatly between infected individuals, older age, and specific comorbidities confer a worse prognosis. Obesity, diabetes mellitus, cardiovascular, respiratory diseases, and cancer are associated with poor outcome [2,3].

Patients with hematological malignancies often experience severe disease and have high case fatality (CFR) and mortality

A full list of author affiliations appears at the end of the paper.

Received: 28 July 2021 Revised: 29 September 2021 Accepted: 5 October 2021

Published online: 1 November 2021 
rates. A meta-analysis of 3240 adult patients with a hematological malignancy reported a risk of death of $34 \%$ [4]. Moreover, in a study of 740 patients with hematological malignancies from Turkey the CFR was significantly higher when compared with age, sex, and comorbidity-matched controls (13.8\% vs $6.8 \%$ ) [5]. In a similar patient group from Italy, Passamonti et al. reported a standardized mortality ratio of 2.04 (95\% Cl 1.77-2.34) compared with the Italian general population with COVID-19 [6].

Chronic lymphocytic leukemia (CLL) is the most frequent adult hematological malignancy in the West, affecting particularly the elderly, with a median age at diagnosis of 72 years [7]. CLL is characterized by an impaired immune capacity due to a profound immune dysregulation reflected by hypogammaglobulinemia, qualitative and quantitative $\mathrm{B}$ - and T-cell defects, CD4 ${ }^{+}$lymphopenia, innate immune dysfunction, and neutropenia [8]. Antileukemic treatments can further weaken the immune response to common pathogens, rendering patients more susceptible to infections [9]. CLL immune defects may hinder the host from effectively controlling SARS-CoV-2 replication. Conversely, while patients with CLL might struggle to eliminate the virus, they may be less prone to a cytokine-induced inflammatory hyperactivation that can lead to acute respiratory distress syndrome, multiorgan damage, and death [10].

Early in the pandemic, reports from hospitalized patients showed that those with hematological malignancies were more susceptible to severe infection from SARS-CoV-2 and had higher CFR compared to the general population [11, 12]. In those studies, CLL was underrepresented and the impact of specific antileukemic treatments was not assessed. In a COVID-19 and CLL-specific cohort of 46 Italian patients, the Campus CLL group reported a CFR of $30.4 \%$ [13]. Following this report, ERIC, the European Research Initiative on CLL, and Campus CLL reported data from 190 patients [14]. Among hospitalized patients, the CFR was $32.5 \%$. Age and number of comorbidities did not impact on overall survival (OS); patients on recent or ongoing treatment were more likely to have milder COVID-19 compared to untreated patients; finally, patients treated with the Bruton's tyrosine kinase inhibitor (BTKi) ibrutinib were less likely to be hospitalized, prompting us to suggest a possible protective effect.

Mato et al. reported the outcomes of 198 patients with COVID19 and CLL mainly from the USA [15]. The overall CFR was 33\%. No difference was observed between treated and untreated patients with regard to infection severity and mortality. Advanced age and specific comorbidities conferred a worse prognosis. BTKi did not show a protective effect; however, in most cases, they were held during the infection.

The ERIC and US cohorts were also analyzed together with a Spanish cohort in a joint effort. The CFR was 30-34\%. Age was the only risk factor of fatality in both cohorts, while the effect of CLL treatment on OS was inconsistent across cohorts. None of the CLLdirected treatments affected OS [16].

As SARS-CoV-2 is still surging across the globe, we significantly expanded our retrospective international multicenter cohort of patients with CLL and COVID-19. We aimed at reassessing the risk factors for COVID-19-related fatality and elucidate further the impact of CLL-directed treatments.

\section{METHODS}

\section{Data collection}

This is a retrospective international multicenter study of the ERIC and the Campus CLL. It represents a continuation of a previous study conducted during the first wave of the pandemic [14]. Investigators at each center updated the information of their previous cases and added any new patients with COVID-19 and CLL/small lymphocytic lymphoma (SLL) or high-count CLL-like monoclonal B-cell lymphocytosis (MBL), a pre-CLL condition, also characterized by inherent immune compromise [17]. The establishment of CLL diagnosis, treatment decisions, review of medical history, and assessment of patient status were performed by the local teams following international guidelines [18]. The study was approved by the institutional ethics committees and data were processed and treated lawfully and fairly in a transparent manner that ensured appropriate security of the personal data, abiding by the General Data Protection Regulation. Informed consent was obtained from all patients that survived the infection. Data collection took place between March 28 and May 22, 2020 (first study) and between December 01, 2020, and March 16, 2021 (current study). Investigators reported all their patients with CLL or related conditions (SLL or MBL) who were diagnosed with COVID-19 from the beginning of the pandemic until the completion of the collection.

Ninety-one institutions participated in the study providing information for a total of 1009 cases. After excluding cases with uncertain diagnosis ('atypical CLL') and those without a qRT-PCR positive test for SARS-CoV-2, we resulted in 941 patients to be studied, 190 of whom were included in the first study. The full list of participating countries is reported in Supplemental Table 1.

Data extracted from the medical records included: baseline demographics; date of CLL diagnosis; IGHV gene somatic hypermutation status; cytogenetic status for chromosomes 11q, 13q 17p, and 12 determined by fluorescence in situ hybridization (at last assessment); TP53 gene mutation status assessed by Sanger sequencing or next-generation sequencing (at last assessment); CLL treatment status; presence, number, and type of comorbidities, date of COVID-19, COVID-19 symptoms, COVID-19 management, treatment, complications, and outcome.

OS was defined as the time from suspected COVID-19 to death or last follow-up date.

In keeping with international practice, patients were deemed to have COVID-19 if a qRT-PCR assay test from a throat or nose swab was positive for SARS-CoV-2.

Severe COVID-19 was defined as hospitalization and need of oxygen or admission into an intensive care unit; nonsevere/mild COVID-19 was defined as confinement at home or hospitalization without need of oxygen.

Patients diagnosed with COVID-19 from the start of the pandemic until June 30, 2020, were considered to belong to the 1st wave of SARS-CoV-2, while patients diagnosed from July 1 st until the completion of data collection were designated to the 2 nd wave. The cut-off was decided based on the disposition of cases in our cohort (Supplemental Fig. 1).

\section{Statistical analysis}

Median (IQR) is used to describe numeric variables while frequencies and percentages are used for categorical. Both univariate and multivariate analyses were carried out, having COVID-19 disease severity, CFR, or OS as outcomes. As potential risk factors, we examined clinico-biological characteristics (age, comorbidities, gender, IGHV gene somatic hypermutation status, TP53 aberrations), COVID-19 disease severity (where appropriate), CLL treatment status (at the time of COVID-19 and in the last 12 months), type of CLL treatment (at the time of COVID-19 and in the last 12 months), and measures taken for the management of COVID-19 (continued as planned, replaced with other, or stopped the treatment). Also, we compared CFR between the two waves. To this end, to reduce the impact of confounding factors, we firstly tested for differences of the above-mentioned risk factors between the two waves. The statistical analyses were carried out in either all patients or subsets of the dataset (only severe, only patients receiving BTKi at the time of COVID-19). In the analyses for CFR, patients who were still under medical care were excluded. The significance level was set to $5 \%$. In post-hoc comparisons, the correction of Bonferroni was used.

For COVID-19 disease severity and CFR, $X^{2}$ test or Fisher's exact test (when necessary) were used for univariate analyses, while logistic regression was used for the multivariate. When necessary, we performed bias reduction techniques to the logistic model estimates by adjusting Firth's logistic regression. Furthermore, for the comparison of CFR between the two waves, age was used as a confounder, since statistically significant older patients were identified in the first wave. For the comparisons of the numeric risk factors between the two waves, independent samples $t$-tests (Student's or Welch's) were conducted. The homogeneity of variance between the two groups was examined through Levene's test. For the categorical risk factors, $X^{2}$ test or Fisher's exact test were used. 
For OS, the log-rank test was used for the univariate analyses, and Cox regression was conducted for the multivariate.

For the multivariate analyses, we performed a two-level variable selection approach. At first, we obtained the statistically significant risk factors through univariate analyses and used them as risk factors for a multivariate model. We further explored the multivariate model by performing backward elimination using $p$ value.

All statistical analyses were conducted using $R$ 4.0.4. We used brglm package [19] for Firth's logistic regression, survival [20], and survminer packages [21] for survival analysis and ggplot2 package [22] for data visualization (Kaplan-Meier curves and the line-plot).

\section{RESULTS}

\section{Patient characteristics}

We included 941 patients with a median age of 69 years (IQR 61-77) at the time of SARS-CoV-2 infection. Most patients were diagnosed with CLL (887, 94.3\%), while 38 and 16 patients were diagnosed with SLL and MBL, respectively. The majority (628/941, $66.7 \%$ ) were male with a median number of comorbidities of 2 . Baseline patient characteristics are shown in Table 1.

Regarding CLL history, 394 patients (41.9\%) had never received treatment for CLL, while 547 (58.1\%) had been treated with at least one line of treatment. Amongst treated patients, 320 (34\%) were on treatment at the time of SARS-CoV-2 infection. The most

Table 1. Patient Characteristics.

\begin{tabular}{|c|c|c|c|}
\hline \multicolumn{2}{|c|}{ Patient characteristics } & Results & Missing \\
\hline \multicolumn{2}{|c|}{$\begin{array}{l}\text { Age at COVID-19, years } \\
\text { (median, IQR) }\end{array}$} & $69(61-77)$ & $0(0 \%)$ \\
\hline \multirow[t]{2}{*}{ Gender } & Female & $313(33.3 \%)$ & \multirow[t]{5}{*}{$0(0 \%)$} \\
\hline & Male & $628(66.7 \%)$ & \\
\hline \multirow[t]{3}{*}{ Diagnosis } & CLL & 887 (94.3\%) & \\
\hline & MBL & $16(1.7 \%)$ & \\
\hline & SLL & $38(4 \%)$ & \\
\hline \multicolumn{2}{|c|}{ Obesity (BMI > 30) } & $151(17.3 \%)$ & $70(7.4 \%)$ \\
\hline \multirow[t]{3}{*}{ Smoking } & Current smoker & $73(8.8 \%)$ & $112(11.9 \%)$ \\
\hline & Ex-smoker & $222(26.8 \%)$ & \\
\hline & Never & $534(64.4 \%)$ & \\
\hline \multicolumn{2}{|c|}{$\begin{array}{l}\text { Hypogammaglobulinemia (lgG < } \\
550 \mathrm{mg} / \mathrm{dL} \text { ) }\end{array}$} & $352(49.5 \%)$ & $230(24.4 \%)$ \\
\hline \multicolumn{2}{|c|}{ CIRS score (median, range) } & $4(0-32)$ & $76(8.1 \%)$ \\
\hline \multicolumn{2}{|c|}{$\begin{array}{l}\mathrm{N} \text { of comorbidities } \\
\text { (median, range) }\end{array}$} & $2(0-11)$ & $0(0 \%)$ \\
\hline \multicolumn{2}{|c|}{ Other respiratory } & $61(6.5 \%)$ & $4(0.4 \%)$ \\
\hline \multicolumn{2}{|l|}{ Asthma } & $21(2.2 \%)$ & $4(0.4 \%)$ \\
\hline \multicolumn{2}{|l|}{ COPD } & $61(6.5 \%)$ & $4(0.4 \%)$ \\
\hline \multicolumn{2}{|c|}{ Cardiac Failure } & $30(3.2 \%)$ & $4(0.4 \%)$ \\
\hline \multicolumn{2}{|c|}{ Arrythmias } & $87(9.3 \%)$ & $4(0.4 \%)$ \\
\hline \multicolumn{2}{|c|}{ Coronary artery disease } & $94(10 \%)$ & $4(0.4 \%)$ \\
\hline \multicolumn{2}{|c|}{ Other cardiovascular } & $83(8.9 \%)$ & $4(0.4 \%)$ \\
\hline \multicolumn{2}{|c|}{ Hypertension } & $440(47 \%)$ & $4(0.4 \%)$ \\
\hline \multicolumn{2}{|l|}{ Diabetes } & $173(18.5 \%)$ & $4(0.4 \%)$ \\
\hline \multicolumn{2}{|c|}{ Chronic renal disease } & $51(5.4 \%)$ & $4(0.4 \%)$ \\
\hline \multicolumn{2}{|c|}{$\begin{array}{l}\text { Other hematological } \\
\text { malignancies }\end{array}$} & $10(1.1 \%)$ & $4(0.4 \%)$ \\
\hline \multicolumn{2}{|c|}{$\begin{array}{l}\text { Other non-hematological } \\
\text { malignancies (excluding skin) }\end{array}$} & $75(8 \%)$ & $4(0.4 \%)$ \\
\hline
\end{tabular}

$I Q R$ interquartile range, CIRS cumulative illness rating scale, COPD chronic obstructive pulmonary disease, CLL chronic lymphocytic leukemia, SLL small lymphocytic lymphoma, MBL monoclonal B lymphocytosis. common treatment category at the time of COVID-19 was BTKi monotherapy $(56.3 \%)$, followed by venetoclax monotherapy (10.7\%) and chemoimmunotherapy (9.1\%). Detailed information about CLL-directed therapy is given in Table 2.

\section{COVID-19 manifestations and management}

The majority of patients $(887 / 941,94.3 \%)$ were symptomatic at the time of documented SARS-COV-2 infection. Fever was the most common COVID-19 related symptom (Supplemental Table 2).

In total, $237 / 941$ (25.3\%) patients were confined at home, while $695 / 941(74.7 \%)$ patients were admitted to the hospital. Of the hospitalized patients, 177 (25.5\%) were admitted to the ICU, 440 (63.3\%) needed oxygen supplementation outside the ICU, and 78 (11.2\%) were hospitalized without need of oxygen (Table 3 ).

Most hospitalized patients received pharmacologic treatment for COVID-19. Corticosteroids were the most commonly used treatment. In most cases (212/320,66.2\%), CLL-directed treatment was stopped during COVID-19 infection (Table 2). Signs of pneumonia were detected in $659 / 874$ patients $(75.4 \%)$. Fortyeight patients (6.2\%) developed venous thromboembolism (40/48 pulmonary embolism) (Table 3 ).

\section{COVID-19 severity}

Age, CIRS score, hypogammaglobulinemia, and specific comorbidities (COPD, coronary artery disease, diabetes, chronic renal disease) were identified as risk factors of severity in the univariate analyses. In the multivariate model, age $(\mathrm{OR}=1.04 ; 95 \% \mathrm{Cl}$ : $1.02-1.06 ; p<0.001)$, hypogammaglobulinemia (OR $=1.69 ; 95 \%$ $\mathrm{Cl}: 1.20-2.38 ; p=0.002)$, and coronary artery disease $(\mathrm{OR}=2.83$; $95 \% \mathrm{Cl}: 1.37-6.61 ; p=0.009)$ remained statistically significant (Supplemental Table 3).

CLL treatment did not affect the severity of COVID-19. No difference was found in terms of severity between patients receiving $B$ TKi at the time of COVID-19 versus untreated patients. Among 179 patients receiving BTKi at the time of COVID-19, age $(\mathrm{OR}=1.04 ; 95 \% \mathrm{Cl}: 1.01-1.08 ; p=0.017)$ and hypogammaglobulinemia $(\mathrm{OR}=2.21 ; 95 \% \mathrm{Cl}: 1.13-4.36 ; p=0.021)$ remained statistically significant predictors in the multivariate analysis (Supplemental Table 4).

\section{Overall survival and risk factors of fatality}

In total, the CFR for all patients was 27.3\% (257/941) (Supplemental Fig. 2). In 647 patients (69\%) the infection resolved, 34 were under medical care at the time of data collection and in 3 the infection outcome was missing (Table 3 ). The vast majority of deaths occurred in patients with severe COVID-19 (236/617). Only 15 deaths occurred amongst 315 patients with nonsevere COVID19 (CFR: 4.8\%).

The baseline characteristics were similar between the two waves, except for age $(p<0.001)$ (Supplementary Tables 5, 6, and 7). The median age in the first wave was 71 years (with $39.9 \%$ older than 75 years) while in the second wave it was 68 years (with $27 \%$ older than 75 years). No difference in CFR was found between the first and second wave of COVID-19 in both all patients or only in patients with severe COVID-19, even after controlling for age ( $p$ $=0.78 ; p=0.16$, respectively) (Supplemental Tables 5 and 6).

In order to avoid collection bias, we restricted the analysis for both infection outcome and OS to patients with severe COVID-19 infection. We also excluded patients who were still under medical care and those missing information about the infection outcome.

The CFR for patients with severe COVID-19 was $38.4 \%$ (Supplemental Fig. 3). Through univariate analyses, age, unmutated IGHV gene somatic hypermutation status, specific comorbidities, and treatment status were observed as statistically significant risk factors for mortality. Age $(\mathrm{OR}=1.04 ; 95 \% \mathrm{Cl}$ : 1.02-1.06; $p<0.001)$, cardiac failure $(\mathrm{OR}=3.82 ; 95 \% \mathrm{Cl}: 1.31-13.94$; $p=0.023)$ and treatment in the last 12 months $(\mathrm{OR}=2.13 ; 95 \% \mathrm{Cl}$ : $1.44-3.20 ; \quad p<0.001)$ remained statistically significant in 
Table 2. CLL-directed treatment.

\begin{tabular}{|c|c|c|c|c|}
\hline Treatment & Category & Number & Percentage & Missing \\
\hline \multirow[t]{2}{*}{ CLL treatment status } & Treated & 547 & $58.1 \%$ & \multirow[t]{2}{*}{$0(0 \%)$} \\
\hline & Untreated & 394 & $41.9 \%$ & \\
\hline \multirow[t]{2}{*}{ Treated status in last 12 months } & Treated & 432 & $46 \%$ & \multirow[t]{2}{*}{$2(0.2 \%)$} \\
\hline & Untreated & 507 & $54 \%$ & \\
\hline \multirow[t]{2}{*}{ Treatment status for CLL at the time of COVID-19 } & Treated & 320 & $34 \%$ & \multirow[t]{2}{*}{$1(0.1 \%)$} \\
\hline & Untreated & 620 & $66 \%$ & \\
\hline \multirow[t]{3}{*}{ Management of CLL treatment } & Continued as planned & 104 & $32.7 \%$ & \multirow[t]{3}{*}{$(0.6 \%)$} \\
\hline & Replaced with other & 2 & $0.6 \%$ & \\
\hline & Stopped & 212 & $66.7 \%$ & \\
\hline \multirow[t]{5}{*}{ Total prior lines of treatment } & 1 & 275 & $50.7 \%$ & \multirow[t]{5}{*}{$5(0.9 \%)$} \\
\hline & 2 & 149 & $27.5 \%$ & \\
\hline & 3 & 60 & $11.1 \%$ & \\
\hline & 4 & 33 & $6.1 \%$ & \\
\hline & $>4$ & 25 & $4.6 \%$ & \\
\hline \multirow[t]{11}{*}{ Treatment at the time of COVID-19 } & BTKi & 179 & $56.3 \%$ & \multirow[t]{11}{*}{$2(0.6 \%)$} \\
\hline & Venetoclax & 34 & $10.7 \%$ & \\
\hline & Venetoclax +Anti-CD20 & 17 & $5.3 \%$ & \\
\hline & PI3K inhibitors & 10 & $3.1 \%$ & \\
\hline & PI3K inhibitors + Anti-CD20 & 3 & $0.9 \%$ & \\
\hline & Anti-CD20 & 8 & $2.5 \%$ & \\
\hline & Chemotherapy & 22 & $6.9 \%$ & \\
\hline & Chemoimmunotherapy & 29 & $9.1 \%$ & \\
\hline & BTKi + Venetoclax & 7 & $2.2 \%$ & \\
\hline & BTKi + Venetoclax + Anti-CD20 & 2 & $0.6 \%$ & \\
\hline & Steroids only & 7 & $2.2 \%$ & \\
\hline
\end{tabular}

PI3K Phosphatidylinositol-3 kinase, BTKi Bruton's tyrosine kinase inhibitor.

\footnotetext{
PI3k Phosphatidylinositol-3 kinase, BTKi Bruton's tyrosine kinase inhibitor.
}

Table 3. COVID-19 management, complications, and outcome.

\begin{tabular}{|c|c|c|c|c|}
\hline & & Number & Percentage & Missing \\
\hline \multirow{4}{*}{$\begin{array}{l}\text { Measures taken for } \\
\text { management of COVID-19 }\end{array}$} & Intensive care & 177 & $19 \%$ & \multirow[t]{4}{*}{$9(1 \%)$} \\
\hline & Hospitalization with need of oxygen & 440 & $47.2 \%$ & \\
\hline & Hospitalization without need of oxygen & 78 & $8.4 \%$ & \\
\hline & Confinement at home only & 237 & $25.4 \%$ & \\
\hline \multirow[t]{2}{*}{ Disease severity } & Severe & 617 & $66.2 \%$ & \multirow[t]{2}{*}{$9(1 \%)$} \\
\hline & Nonsevere & 315 & $33.8 \%$ & \\
\hline Hospitalization & Home & 237 & $25.4 \%$ & $9(1 \%)$ \\
\hline \multicolumn{2}{|l|}{ Antiviral } & 266 & $35.7 \%$ & $195(20.7 \%)$ \\
\hline \multicolumn{2}{|l|}{ Hydroxycloroquine or similar } & 203 & $27.1 \%$ & $192(20.4 \%)$ \\
\hline \multicolumn{2}{|l|}{ Azithromycin } & 265 & $35.9 \%$ & $202(21.5 \%)$ \\
\hline \multicolumn{2}{|l|}{ Steroids } & 514 & $61.3 \%$ & $103(10.9 \%)$ \\
\hline $\begin{array}{l}\text { Complications of COVID-19 } \\
\text { infection }\end{array}$ & VTE & 48 & $6.2 \%$ & $167(17.7 \%)$ \\
\hline \multicolumn{2}{|l|}{ PE (only for 48 with VTE) } & 40 & $87 \%$ & $2(4.2 \%)$ \\
\hline \multirow[t]{3}{*}{ Infection outcome } & Resolution & 647 & $69 \%$ & \multirow[t]{3}{*}{$3(0.3 \%)$} \\
\hline & Still under medical care & 34 & $3.6 \%$ & \\
\hline & Death & 257 & $27.4 \%$ & \\
\hline
\end{tabular}

DIC disseminated intravascular coagulation, VTE venous thromboembolism, PE pulmonary embolism. 
Table 4. Risk factors of infection outcome for patients with severe COVID-19 $(n=594)$.

\begin{tabular}{|c|c|c|c|c|c|}
\hline \multirow[t]{2}{*}{ Risk factor } & \multirow[t]{2}{*}{ Categories } & \multicolumn{2}{|c|}{ Infection outcome } & \multirow[t]{2}{*}{$\mathbf{R R}$} & \multirow[t]{2}{*}{$p$ value } \\
\hline & & Resolution & Death & & \\
\hline \multirow[t]{2}{*}{ Age } & $\geq 65$ & $240(57.1 \%)$ & $180(42.9 \%)$ & \multirow[t]{2}{*}{1.33} & \multirow[t]{2}{*}{0.02} \\
\hline & $<65$ & $118(67.8 \%)$ & $56(32.2 \%)$ & & \\
\hline \multirow[t]{2}{*}{ Age } & $\geq 75$ & $104(47.5 \%)$ & $115(52.5 \%)$ & \multirow[t]{2}{*}{1.63} & \multirow[t]{2}{*}{$<0.001$} \\
\hline & $<75$ & $254(67.7 \%)$ & $121(32.3 \%)$ & & \\
\hline \multirow[t]{2}{*}{ Gender } & Male & $117(60.9 \%)$ & $75(39.1 \%)$ & \multirow[t]{2}{*}{0.98} & \multirow[t]{2}{*}{0.89} \\
\hline & Female & $241(60 \%)$ & $161(40 \%)$ & & \\
\hline \multirow[t]{2}{*}{ IGHV gene somatic hypermutation status } & Mutated $^{a}$ & $91(66.4 \%)$ & $46(33.6 \%)$ & \multirow[t]{2}{*}{0.70} & \multirow[t]{2}{*}{0.017} \\
\hline & Unmutated $^{\mathrm{b}}$ & 91 (52.3\%) & $83(47.7 \%)$ & & \\
\hline del(13q) (last assessment) & Negative & $113(56.5 \%)$ & $87(43.5 \%)$ & 1.14 & 0.37 \\
\hline & Positive & $103(61.7 \%)$ & $64(38.3 \%)$ & & \\
\hline del(11q) (last assessment) & Negative & $185(60.3 \%)$ & $122(39.7 \%)$ & 0.83 & 0.3 \\
\hline & Positive & $34(52.3 \%)$ & $31(47.7 \%)$ & & \\
\hline trisomy 12 (last assessment) & Negative & $176(60.5 \%)$ & $115(39.5 \%)$ & 0.87 & 0.45 \\
\hline & Positive & $36(54.5 \%)$ & $30(45.5 \%)$ & & \\
\hline del(17p) (last assessment) & Negative & $206(59.7 \%)$ & $139(40.3 \%)$ & 0.75 & 0.09 \\
\hline & Positive & $23(46 \%)$ & $27(54 \%)$ & & \\
\hline TP53 mutation status & Mutated & $18(48.6 \%)$ & $19(51.4 \%)$ & 1.23 & 0.37 \\
\hline & Unmutated & $137(58.1 \%)$ & $99(41.9 \%)$ & & \\
\hline del(17p) positive and/or TP53 mutation & Yes & $33(50 \%)$ & $33(50 \%)$ & 1.2 & 0.29 \\
\hline & No & $130(58.3 \%)$ & $93(41.7 \%)$ & & \\
\hline CIRS score & $\leq 6$ & $242(65.4 \%)$ & $128(34.6 \%)$ & 0.68 & $<0.001$ \\
\hline & $>6$ & $87(49.4 \%)$ & $89(50.6 \%)$ & & \\
\hline Other respiratory & Yes & $24(60 \%)$ & $16(40 \%)$ & 1 & $>0.99$ \\
\hline & No & $332(60.1 \%)$ & 220 (39.9\%) & & \\
\hline Asthma & Yes & $10(66.7 \%)$ & $5(33.3 \%)$ & 0.83 & 0.8 \\
\hline & No & $346(60 \%)$ & $231(40 \%)$ & & \\
\hline COPD & Yes & $22(47.8 \%)$ & $24(52.2 \%)$ & 1.35 & 0.11 \\
\hline & No & $334(61.2 \%)$ & $212(38.8 \%)$ & & \\
\hline Cardiac Failure & Yes & $5(25 \%)$ & $15(75 \%)$ & 1.94 & 0.002 \\
\hline & No & $351(61.4 \%)$ & $221(38.6 \%)$ & & \\
\hline Arrythmias & Yes & $33(57.9 \%)$ & $24(42.1 \%)$ & 1.06 & 0.83 \\
\hline & No & $323(60.4 \%)$ & 212(39.6) & & \\
\hline Coronary artery disease & Yes & $32(44.4 \%)$ & $40(55.6 \%)$ & 1.47 & 0.006 \\
\hline & No & $324(62.3 \%)$ & $196(37.7 \%)$ & & \\
\hline Other cardiovascular & Yes & $32(54.2 \%)$ & $27(45.8 \%)$ & 1.17 & 0.4 \\
\hline & No & $324(60.8 \%)$ & 209 (39.2\%) & & \\
\hline Hypertension & Yes & $167(56.8 \%)$ & $127(43.2 \%)$ & 1.18 & 0.12 \\
\hline & No & 189 (63.4\%) & 109 (36.6\%) & & \\
\hline Diabetes & Yes & $80(64 \%)$ & 45 (36\%) & 0.88 & 0.37 \\
\hline & No & $276(59.1 \%)$ & $191(40.9 \%)$ & & \\
\hline Chronic renal disease & Yes & $15(40.5 \%)$ & $22(59.5 \%)$ & 1.54 & 0.019 \\
\hline & No & $341(61.4 \%)$ & $214(38.6 \%)$ & & \\
\hline Other hematological malignancies & Yes & $5(55.6 \%)$ & $4(44.4 \%)$ & 1.16 & 0.75 \\
\hline & No & $351(60.2 \%)$ & $232(39.8 \%)$ & & \\
\hline Other non-hematological malignancies (excluding skin) & Yes & $27(61.4 \%)$ & $17(38.6 \%)$ & 0.97 & 0.99 \\
\hline & No & $329(60 \%)$ & $219(40 \%)$ & & \\
\hline Obesity (BMI>30) & Yes & $57(61.3 \%)$ & $36(38.7 \%)$ & 0.99 & $>0.99$ \\
\hline & No & $280(61.1 \%)$ & $178(38.9 \%)$ & & \\
\hline Smoking & Current smoker & $25(59.5 \%)$ & $17(40.5 \%)$ & & 0.58 \\
\hline & Ex-smoker & $82(57.7 \%)$ & $60(42.3 \%)$ & & \\
\hline & Never & $212(62.7 \%)$ & $126(37.3 \%)$ & & \\
\hline Hypogammaglobulinemia (lgG <550 mg/dL) & Present & $133(55.6 \%)$ & $106(44.4 \%)$ & 1.33 & 0.017 \\
\hline & Absent & $146(67 \%)$ & $72(33 \%)$ & & \\
\hline CLL treatment status & Treated & $168(49.9 \%)$ & $169(50.1 \%)$ & 1.92 & $<0.001$ \\
\hline & Untreated & $190(73.9 \%)$ & $67(26.1 \%)$ & & \\
\hline CLL treatment status at the time of COVID-19 & Treated & $94(47.7 \%)$ & $103(52.3 \%)$ & 1.56 & $<0.001$ \\
\hline & Untreated & $263(66.4 \%)$ & $133(33.6 \%)$ & & \\
\hline
\end{tabular}


Table 4 continued

\begin{tabular}{|c|c|c|c|c|c|c|}
\hline \multirow[t]{2}{*}{ Risk factor } & \multirow{2}{*}{\multicolumn{2}{|c|}{ Categories }} & \multicolumn{2}{|c|}{ Infection outcome } & \multirow[t]{2}{*}{ RR } & \multirow[t]{2}{*}{$p$ value } \\
\hline & & & Resolution & Death & & \\
\hline \multirow[t]{2}{*}{ Treated in last 12 months } & \multicolumn{2}{|l|}{ Yes } & $132(48.4 \%)$ & $141(51.6 \%)$ & 1.42 & 0.002 \\
\hline & \multicolumn{2}{|l|}{ No } & $116(63.7 \%)$ & $66(36.3 \%)$ & & \\
\hline Antiviral & \multicolumn{2}{|l|}{ No } & $177(66 \%)$ & $91(34 \%)$ & & \\
\hline Hydroxycloroquine or similar & \multicolumn{2}{|l|}{ Yes } & $116(67.1 \%)$ & 57 (32.9\%) & 0.92 & 0.61 \\
\hline Azithromycin & \multicolumn{2}{|l|}{ No } & $187(63.6 \%)$ & $107(36.4 \%)$ & & \\
\hline \multirow[t]{2}{*}{ Steroids } & \multicolumn{2}{|l|}{ Yes } & $264(63 \%)$ & $155(37 \%)$ & 1.04 & 0.88 \\
\hline & \multicolumn{2}{|l|}{ No } & $67(64.4 \%)$ & $37(35.6 \%)$ & & \\
\hline \multirow[t]{2}{*}{ Anti-IL6/IL6R } & \multicolumn{2}{|l|}{ Yes } & $55(66.3 \%)$ & $28(33.7 \%)$ & 0.95 & 0.87 \\
\hline & \multicolumn{2}{|l|}{ NO } & $257(64.6 \%)$ & $141(35.4 \%)$ & & \\
\hline Country & \multicolumn{2}{|l|}{ Italy } & $108(61.7 \%)$ & $67(38.3 \%)$ & & 0.09 \\
\hline Risk factor & \multicolumn{3}{|c|}{ OR } & $95 \% \mathrm{Cl}$ & & $p$ value \\
\hline Age (years) & \multicolumn{3}{|c|}{1.04} & $1.02-1.06$ & & $<0.001$ \\
\hline Cardiac failure (YES vs NO) & \multicolumn{3}{|c|}{3.82} & $1.31-13.94$ & & 0.023 \\
\hline Treated in last 12 months (Treated vs Untreated) & \multicolumn{3}{|c|}{2.13} & $1.44-3.20$ & & $<0.001$ \\
\hline
\end{tabular}

multivariate analysis (Table 4). We performed the same analysis including only the patients with severe COVID-19 that were reported from centers that participated in both studies. In the multivariate analysis, age and CLL treatment status remained statistically significant risk factors (Supplemental Table 8).

Univariate and multivariate analyses of risk factors of OS are outlined in Table 5. Older age (HR=1.03; 95\% Cl: $1.02-1.04 ; p<$ $0.001)$, cardiac failure ( $\mathrm{HR}=1.79 ; 95 \% \mathrm{Cl}: 1.04-3.07 ; p=0.035)$ and treatment status $(\mathrm{HR}=0.54 ; 95 \% \mathrm{Cl}: 0.41-0.72 ; p<0.001)$ appeared as statistically significant risk factors of OS in the multivariate analysis.

Comparison of OS between treated and untreated patients is depicted graphically in Fig. 1.

\section{CLL-directed treatment and COVID-19 outcome}

We assessed the impact of specific treatment categories on COVID-19 outcome. Initially, we compared patients receiving BTKi at the time of SARS-COV-2 infection $(n=169)$ with patients receiving venetoclax at the same time $(n=31)$ and patients who had received chemoimmunotherapy in the last 12 months $(n=$ 85). Then, in a second comparison, we kept the first two categories and replaced the third one with patients who had received any anti-CD20-based therapy (alone or in combination) in the last 12 months $(n=128)$. No statistically significant difference between the groups was noted in either analysis. Nonetheless, treated patients (in any treatment category) had a worse OS when compared to the untreated ones $(p<0.001)$ (Fig. 2 and Supplemental Fig. 4).

One hundred and ten patients with severe COVID-19 were being treated with BTKi monotherapy at the time of COVID-19. Thirty patients continued the BTKi treatment, while in 78 cases physicians chose to hold the drug. Patients who continued BTKi treatment did not have a statistically better outcome than those who discontinued the treatment $(p=0.08)$, while both groups had a worse outcome compared with untreated patients $(p<0.001)$ (Table 6$)$.

\section{DISCUSSION}

We here present the largest cohort published to date of patients with CLL and COVID-19. In this homogeneous disease-specific series, age, history of cardiac failure, and CLL treatment were the main risk factors for a dismal outcome.

In our study, the finding that older patients with CLL often have severe disease, higher CFRs, and lower survival time is in line with data derived from the general population, where COVID-19related fatality and time to death positively correlated with age $[3,23,24]$. Relevant to add, similar results have been reported in a US study by Mato et al. showing that patients with CLL older than 75 years had a worse OS [15]. Older patients were also more likely to die in our first cohort, albeit without reaching statistical signifigance, which can be attributed to the smaller sample.

In our original cohort including patients from the first wave of SARS-CoV-2 [14], higher CIRS score and certain comorbidities were linked with increased mortality, whereas in this updated cohort only cardiac failure remained a statistically significant comorbidity in the multivariate analysis. OS was unaffected by diabetes, respiratory diseases, and obesity, variables conferring a worse prognosis in COVID-19 patients when analyzed in the general population [2, 3]. Nonetheless, some caution is warranted regarding the precise impact of specific comorbidities on COVID-19 outcome in patients with CLL since information on their severity was not collected systematically.

In this updated cohort, patients treated for CLL had a worse OS than untreated patients, while in both the report by Mato et al (198 patients, end point: outcome) and our previous report (190 patients, end point: severity), no difference was found between treated and untreated patients, probably due to the lower number of cases $[14,15]$. Of note, when our previous cohort was combined with the Spanish cohort (281 patients), untreated patients had better OS than treated ones [16]. Thus, these apparent discrepancies may simply reflect the larger sample of our current analysis that allowed to identify a difference between the 
untreated and the treated, likely more immunosuppressed, patients.

Untreated patients had a better outcome also when compared separately with patients in different treatment categories (BTKi, venetoclax, chemoimmunotherapy, or anti-CD20 containing regimens). Patients who continued BTKi treatment during COVID-19 infection did not appear to fare worse than those who stopped the drug with a potential benefit albeit not statistically significant, suggesting that BTKi continuation definitely is not harming patients and may potentially benefit them by preventing respiratory failure and death or by keeping CLL under control [25]. This is in line with the current recommendations indicating no need to hold BTKi at the time of a confirmed SARS-CoV-2 infection [26].

Patients treated with anti-CD20 antibodies, alone or in combination, had worse OS than untreated patients. This finding suggests that such treatment likely renders patients with CLL more susceptible to succumb to COVID-19 infection. That said, the small number of patients treated with the combination of antiCD20 antibodies with novel agents in the present cohort hinders firm conclusions from being drawn regarding its precise impact on COVID-19 outcome.

The CFR in the entire cohort was $27.4 \%$ and increased to $38.4 \%$ among patients with severe COVID-19. This indicates a remarkable consistency among different studies in patients with CLL and COVID-19 as well as in the different waves of the pandemic, confirming the unabated aggressiveness of the disease with time [14-16]. In the general population, the CFR in hospitalized patients appears lower compared to our cohort: indicatively, a metaanalysis of COVID-19 patients found a fatality rate of $17.1 \%$ and $40.5 \%$ for hospitalized and critically ill patients, respectively [27]. Similarly, in-hospital mortality of patients older than 75 years in a US study was $20.5 \%$ [28]. Nevertheless, the caveats of cross-study comparisons, the retrospective nature of our cohort, and the lack of a control group hinder any generalization about the true impact of CLL in COVID-19-related mortality.

In the updated cohort, CLL-directed treatment was associated with a worse outcome. However, it had no impact on COVID-19 severity in either the orginal or the updated cohort. We suspect that investigators were more likely to be informed about patients on CLL-specific treatment with mild COVID-19 symptoms than untreated patients, since the former were followed up more closely and probably sought immediate guidance: thus, we may have missed more asymptomatic untreated patients.

Dexamethasone and tocilizumab remain the only treatments that showed a mortality benefit in patients with COVID-19 [29, 30]. The impact of COVID-19 treatments in our cohort was not among the objectives, with information on the administered treatment for COVID-19 missing at least in part. Nonetheless, we observed that different types of therapies against COVID-19 did not affect the CFR.

We acknowledge certain limitations in our study. As already mentioned, hospitalized and/or symptomatic patients were more likely to be captured in our cohort, while we probably missed some asymptomatic or mildly symptomatic patients who avoided undergoing testing and/or contacting their physician, especially during the first wave, when COVID-19 testing was not the rule. To avoid the caveats of a retrospective study and the lack of a control group in overestimating the mortality, we restricted the analysis to patients with severe disease and avoided broad generalizations about prevalence, morbidity, and mortality. Taking into account the multicenter, international design of the study, some heterogeneity in our patient population is to be expected as we included patients from different countries, diagnosed with COVID-19 during different waves of the pandemic and managed differently. Along this line, mirroring the course of the pandemic, some countries like Italy and Spain were overrepresented in the first wave, while the second wave featured more patients from other countries
Table 5. Risk factors of OS.

\begin{tabular}{|c|c|c|c|}
\hline \multicolumn{3}{|l|}{ Risk factor } & $p$ value \\
\hline \multicolumn{3}{|l|}{ Age $(\geq 65$ vs $<65)$} & 0.01 \\
\hline \multicolumn{3}{|l|}{ Age $(\geq 75$ vs $<75)$} & $<0.001$ \\
\hline \multicolumn{3}{|l|}{ Gender (Male vs Female) } & 0.56 \\
\hline \multicolumn{3}{|c|}{$\begin{array}{l}\text { IGHV gene somatic hypermutation status (unmutated }{ }^{\mathrm{a}} \text { vs } \\
\text { mutated }^{\mathrm{b}} \text { ) }\end{array}$} & 0.01 \\
\hline \multicolumn{3}{|c|}{ del(13q) (last assessment) (positive vs negative) } & 0.46 \\
\hline \multicolumn{3}{|c|}{ del(11q) (last assessment) (positive vs negative) } & 0.33 \\
\hline \multicolumn{3}{|c|}{ trisomy 12 (last assessment) (positive vs negative) } & 0.59 \\
\hline \multicolumn{3}{|c|}{ del(17p) (last assessment) (positive vs negative) } & 0.02 \\
\hline \multicolumn{3}{|c|}{ TP53 mutation status (unmutated vs mutated) } & 0.18 \\
\hline \multicolumn{3}{|c|}{ del(17p) positive and/or TP53 mutation (yes vs no) } & 0.1 \\
\hline \multicolumn{3}{|l|}{ CIRS score (> 6 vs $\leq 6)$} & $<0.001$ \\
\hline \multicolumn{3}{|l|}{ Other respiratory (YES vs NO) } & 0.82 \\
\hline \multicolumn{3}{|l|}{ Asthma (yes vs no) } & 0.49 \\
\hline \multicolumn{3}{|l|}{ COPD (yes vs no) } & 0.12 \\
\hline \multicolumn{3}{|l|}{ Cardiac Failure (yes vs no) } & $<0.001$ \\
\hline \multicolumn{3}{|l|}{ Arrythmias (yes vs no) } & 0.38 \\
\hline \multicolumn{3}{|l|}{ Coronary artery disease (yes vs no) } & 0.06 \\
\hline \multicolumn{3}{|l|}{ Other cardiovascular (yes vs no) } & 0.16 \\
\hline \multicolumn{3}{|l|}{ Hypertension (yes vs no) } & 0.1 \\
\hline \multicolumn{3}{|l|}{ Diabetes (yes vs no) } & 0.52 \\
\hline \multicolumn{3}{|l|}{ Chronic renal disease (yes vs no) } & 0.02 \\
\hline \multicolumn{3}{|c|}{ Other hematological malignancies (yes vs no) } & 0.91 \\
\hline \multicolumn{3}{|c|}{ Other non-hematological malignancies (yes vs no) } & 0.87 \\
\hline \multicolumn{3}{|l|}{ Obesity (BMl>30) (yes vs no) } & 0.98 \\
\hline \multicolumn{3}{|l|}{ Smoking } & 0.8 \\
\hline \multicolumn{3}{|c|}{$\begin{array}{l}\text { Hypogammaglobulinemia (lgG }<550 \mathrm{mg} / \mathrm{dL} \text { ) (present vs } \\
\text { absent) }\end{array}$} & 0.08 \\
\hline \multicolumn{3}{|c|}{ CLL treatment status (untreated vs treated) } & $<0.001$ \\
\hline \multicolumn{3}{|c|}{ CLL treatment during COVID-19 (treated vs untreated) } & $<0.001$ \\
\hline \multicolumn{3}{|c|}{ Treated in last 12 months (treated vs untreated) } & 0.01 \\
\hline Multivariate analysis & & & \\
\hline Risk factor & HR & $95 \% \mathrm{Cl}$ & \\
\hline Age (years) & 1.03 & $1.02-1.04$ & $<0.001$ \\
\hline Cardiac failure (yes vs no) & 1.79 & $1.04-3.07$ & 0.04 \\
\hline $\begin{array}{l}\text { CLL treatment status (untreated vs } \\
\text { treated) }\end{array}$ & 0.54 & $0.41-0.72$ & $<0.001$ \\
\hline
\end{tabular}

IGHV immunoglobulin heavy variable, CIRS cumulative illness rating scale, COPD chronic obstructive pulmonary disease.

aUnmutated: $\geq 98 \%$ germline identity.

${ }^{\text {b}}$ Mutated: $<98 \%$ germline identity.

(e.g., Czech Republic and Greece). Finally, we recognize that differences in the quality of health care and weather could influence our results. Indeed, only a small number of cases were diagnosed during the summer and none of the patients in our cohort was vaccinated for SARS-CoV-2. In addition, the lack of any difference in CFRs between the two waves illustrates that CLL and treatment-related immunosuppression may overshadow other risk factors of outcome.

Taken together, our findings suggest that in patients with CLL and COVID-19, older age confers a worse prognosis, with increased mortality. Untreated patients had a better chance of survival than those on treatment or recently treated. 


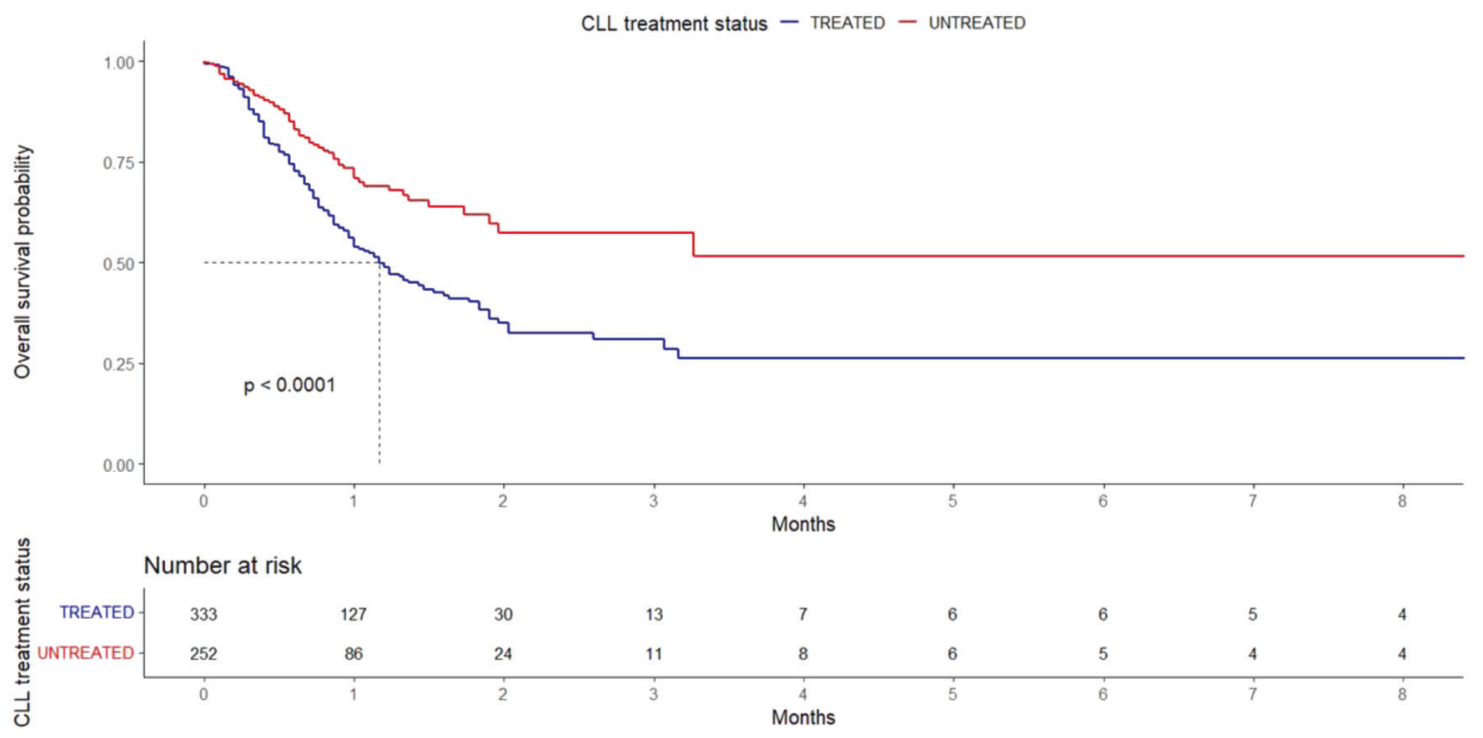

Fig. 1 Overall survival in patients with severe COVID-19. Overall survival comparison between treated and untreated patients with CLL and severe COVID-19.
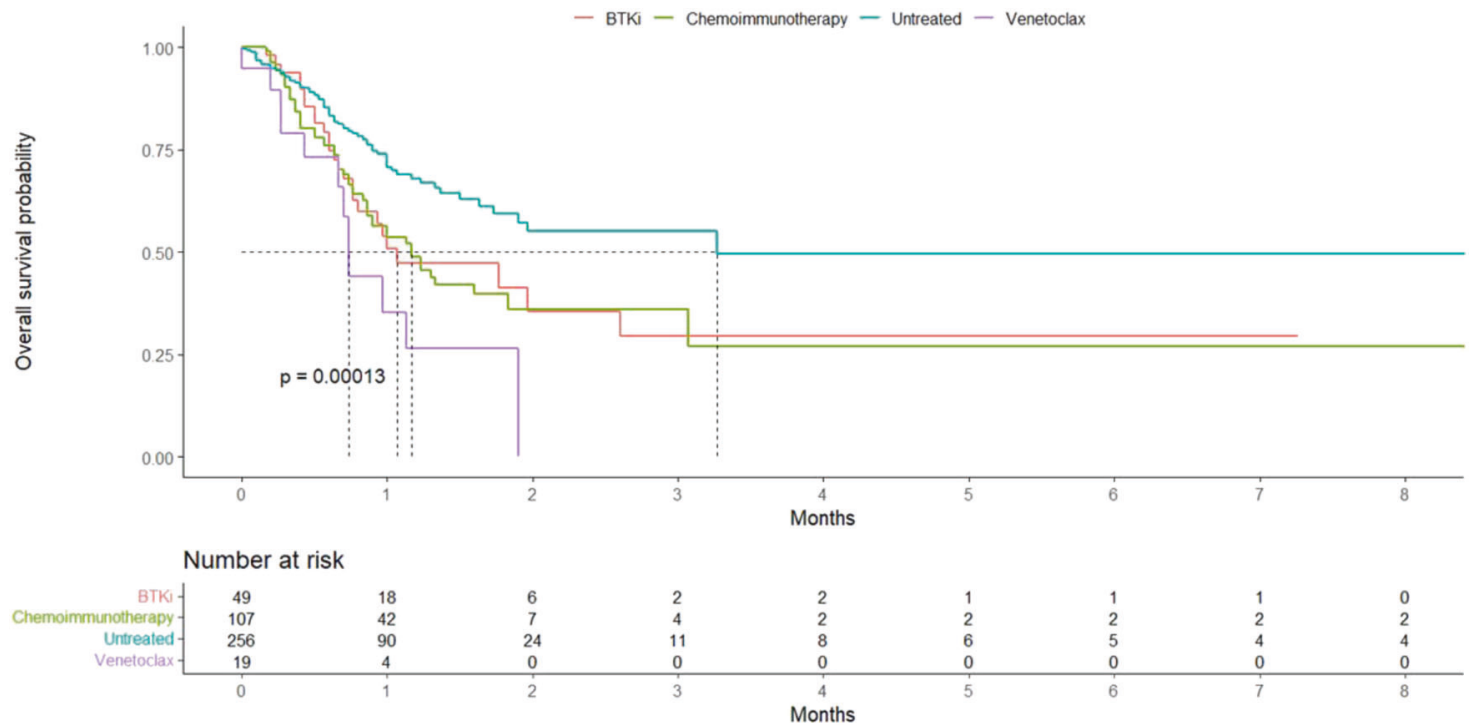

Fig. 2 Overall survival in patients with severe COVID-19 according to treatment. Overall survival comparisons between patients treated with BTKi (at time of COVID-19), Venetoclax (at time of COVID-19), Chemoimmunotherapy in last 12 months, and Untreated.

Table 6. Outcome in patients with severe COVID-19.

\begin{tabular}{llll} 
Category & \multicolumn{2}{l}{ Infection outcome } & p value \\
& Resolution & Death & \\
\hline Untreated & $190(73.9 \%)$ & $67(26.1 \%)$ & $<0.001$ \\
\hline Continued BTKi & $20(66.7 \%)$ & $10(33.3 \%)$ & \\
\hline Stopped BTKi & $36(46.2 \%)$ & $42(53.8 \%)$ & \\
\hline
\end{tabular}

No statistically significant difference between continued and stopped $(p=0.08)$.

BTKi Bruton's tyrosine kinase inhibitor.

\section{CODE AVAILABILITY}

All data created during this study are included in the manuscript and supporting material. The code for biostatistical analysis is available in the following GitHub repository: https://github.com/gkarakatsoulis/COVID-19-in-CLL-patients.

\section{REFERENCES}

1. Zhu N, Zhang D, Wang W, Li X, Yang B, Song J, et al. A novel coronavirus from patients with pneumonia in China, 2019. N Engl J Med. 2020;382:727-33.

2. Harrison SL, Fazio-Eynullayeva E, Lane DA, Underhill P, Lip GYH. Comorbidities associated with mortality in 31,461 adults with COVID-19 in the United States: a federated electronic medical record analysis. PLoS Med. 2020;17:e1003321.

3. Williamson EJ, Walker AJ, Bhaskaran K, Bacon S, Bates C, Morton CE, et al. Factors associated with COVID-19-related death using OpenSAFELY. Nature. 2020;584:430-6.

4. Vijenthira A, Gong IY, Fox TA, Booth S, Cook G, Fattizzo B, et al. Outcomes of patients with hematologic malignancies and COVID-19: a systematic review and meta-analysis of 3377 patients. Blood. 2020;136:2881-92.

5. Yigenoglu TN, Ata N, Altuntas F, Basci S, Dal MS, Korkmaz S, et al. The outcome of COVID-19 in patients with hematological malignancy. J Med Virol. 2021;93:1099-104.

6. Passamonti F, Cattaneo C, Arcaini L, Bruna R, Cavo M, Merli F, et al. Clinical characteristics and risk factors associated with COVID-19 severity in patients with haematological malignancies in Italy: a retrospective, multicentre, cohort study. Lancet Haematol. 2020;7:e737-e45. 
7. Smith A, Howell D, Patmore R, Jack A, Roman E. Incidence of haematological malignancy by sub-type: a report from the Haematological Malignancy Research Network. Br J Cancer. 2011;105:1684-92.

8. Forconi $F$, Moss P. Perturbation of the normal immune system in patients with CLL. Blood. 2015;126:573-81.

9. Tadmor T, Welslau M, Hus I. A review of the infection pathogenesis and prophylaxis recommendations in patients with chronic lymphocytic leukemia. Expert Rev Hematol. 2018;11:57-70.

10. England JT, Abdulla A, Biggs CM, Lee AYY, Hay KA, Hoiland RL, et al. Weathering the COVID-19 storm: Lessons from hematologic cytokine syndromes. Blood Rev. 2021;45:100707.

11. Martin-Moro F, Marquet J, Piris M, Michael BM, Saez AJ, Corona M, et al. Survival study of hospitalised patients with concurrent COVID-19 and haematological malignancies. Br J Haematol. 2020;190:e16-e20.

12. He W, Chen L, Chen L, Yuan G, Fang Y, Chen W, et al. COVID-19 in persons with haematological cancers. Leukemia. 2020;34:1637-45.

13. Cuneo A, Scarfo L, Reda G, Varettoni M, Quaglia FM, Marchetti M, et al. Chronic lymphocytic leukemia management in Italy during the COVID-19 pandemic: a Campus CLL report. Blood. 2020;136:763-6.

14. Scarfo L, Chatzikonstantinou T, Rigolin GM, Quaresmini G, Motta M, Vitale C, et al. COVID-19 severity and mortality in patients with chronic lymphocytic leukemia: a joint study by ERIC, the European Research Initiative on CLL, and CLL Campus. Leukemia. 2020;34:2354-63.

15. Mato AR, Roeker LE, Lamanna N, Allan JN, Leslie L, Pagel JM, et al. Outcomes of COVID-19 in patients with CLL: a multicenter international experience. Blood. 2020;136:1134-43.

16. Roeker LE, Scarfo L, Chatzikonstantinou T, Abrisqueta P, Eyre TA, Cordoba R, et al. Worldwide examination of patients with CLL hospitalized for COVID-19. Blood 2020;136 Supplement 1:45-9.

17. Rawstron AC, Shanafelt T, Lanasa MC, Landgren O, Hanson C, Orfao A, et al. Different biology and clinical outcome according to the absolute numbers of clonal B-cells in monoclonal B-cell lymphocytosis (MBL). Cytom B Clin Cytom. 2010;78 Suppl 1:S19-23.

18. Hallek M, Cheson BD, Catovsky D, Caligaris-Cappio F, Dighiero G, Dohner $\mathrm{H}$, et al. iwCLL guidelines for diagnosis, indications for treatment, response assessment, and supportive management of CLL. Blood 2018;131:2745-60.

19. Kosmidis I. brglm: bias reduction in binary-response generalized linear models. $R$ package version 0.7.2. $2021 \mathrm{https}: / /$ cran.r-project.org/package=brglm.

20. Therneau MT, Grambsch MP. Modeling survival data: extending the cox model. New York: Springer; 2000.

21. Kassambara A, Kosinski M, Biecek P. survminer: Drawing Survival Curves using 'ggplot2'. R package version 0.4.9. 2021. https://CRAN.Rproject.org/package= survminer.

22. Wickham H. ggplot2: elegant graphics for data analysis. New York: SpringerVerlag; 2016.

23. Wu Z, McGoogan JM. Characteristics of and important lessons from the coronavirus disease 2019 (COVID-19) outbreak in China: summary of a report of 72314 cases from the Chinese Center for Disease Control and Prevention. JAMA. 2020;323:1239-42.

24. Richardson S, Hirsch JS, Narasimhan M, Crawford JM, McGinn T, Davidson KW, et al. Presenting characteristics, comorbidities, and outcomes among 5700 patients hospitalized with COVID-19 in the New York city area. JAMA 2020;323:2052-9.

25. Treon SP, Castillo JJ, Skarbnik AP, Soumerai JD, Ghobrial IM, Guerrera ML, et al. The BTK inhibitor ibrutinib may protect against pulmonary injury in COVID-19infected patients. Blood. 2020;135:1912-5.

26. American Society of Hematology (ASH). COVID-19 and CLL: frequently asked questions. ASH; 2021. https://www.hematology.org/covid-19/covid-and-cll.

27. Macedo A, Goncalves N, Febra C. COVID-19 fatality rates in hospitalized patients: systematic review and meta-analysis. Ann Epidemiol. 2021;57:14-21.

28. Finelli L, Gupta V, Petigara T, Yu K, Bauer KA, Puzniak LA. Mortality among US patients hospitalized with SARS-CoV-2 infection in 2020. JAMA Netw Open. 2021;4:e216556.

29. Investigators R-C, Gordon AC, Mouncey PR, Al-Beidh F, Rowan KM, Nichol AD, et al. Interleukin-6 receptor antagonists in critically III patients with Covid-19. N Engl J Med. 2021;384:1491-502.

30. Siemieniuk RA, Bartoszko JJ, Ge L, Zeraatkar D, Izcovich A, Kum E, et al. Drug treatments for covid-19: living systematic review and network meta-analysis. BMJ. 2020;370:m2980.

\section{ACKNOWLEDGEMENTS}

We thank Salma El Ashwah and Marwa O. Elmaria for helping with data collection. This project was supported in part by the "Greece versus Corona" Action of the General Secretariat of Research and Innovation of Greece; "Impact of immune-FRAgilities on
COVID-19 susceptibility and severity" Fondazione Veronesi (Project ID 1852164). RG-S is supported by an Ayuda para la promoción de empleo joven e implantación de la garantía juvenil en I+D+i from Ministerio de Economía, Industria y competitividad, Agencia Estatal de Investigación and Fondo Social Europeo (PEJ2018-004520-A).

\section{AUTHOR CONTRIBUTIONS}

TC, AK and LS collected data, coordinated the study, wrote the paper, and performed the analysis, GK wrote the paper and performed the analysis, DAl, AAC, MA, DAn, MB, PB, DB, ACa, SC, RC, J-GC, CC-G, LDP, MRDP, GDP, CD, MDi, DD, MD, ME, BEi, SE-A, AE, BEs, LF, AF, MF, HF, MFü, JAG-M, RG-S, MG, EG, AG, MGDS, OG, YKH, YH, JAH-R, TH, II, GI, OJ, AJ, OBK, EK, LKK, APK, SK, JL, DL, LL, M-DL, EL, AL-G, LM, RMa, MMar, JM, MMat, FRM, IM, FM, MMor, MMot, TM, RMu, CUN, RNR, JO, LO, MP, MAP, IP, VMP, FMQ, GQ, KQ, GR, GMR, RR, GS, AS, MŠi, MŠp, PS, OS, NS, TT, DTR, SHT, LT, EVDS, MVG, RVK, MV, AV, CV, EWS, TW, LYSS, MY, MC, AR, EM, RF, ACu collected data and contributed to interpretation and manuscript editing, KS and PG designed and coordinated the study, and wrote the paper that was approved by all the authors.

\section{COMPETING INTERESTS}

LS received advisory boards fees from AbbVie and Janssen; educational activity AstraZeneca. DAl received funding to attend symposia from Gilead and Bayer. MA received advisory boards fees from AbbVie, AstraZeneca, and Janssen-Cilag; travel support from AbbVie, and Novartis. DAn received honoraria from AbbVie, Janssen, and Roche. PB has received honoraria from Abbvie, Gilead and Janssen and research funding from Gilead. RC received speaker fees from Roche, Janssen, AbbVie, AstraZeneca, Celgene, BMS, Kite, and Takeda; received advisory board fees from Janssen, AbbVie, Celgene, BMS, Kite, Takeda, Incyte, Kyowa-Kirin, and ADCT; received travel and accommodation expenses from Roche, Janssen, AbbVie, Celgene, BMS, Kite, Takeda, and Pfizer; research grant from Pfizer. MD received honoraria for advisory board and research support from AstraZeneca, AbbVie, Roche, Gilead, and Janssen. BEi received consulting or advisory boards fees from Janssen, Roche, Novartis, AbbVie, Gilead, Celgene, ArQule, AstraZeneca, Oxford Biomedica (UK), and BeiGene; speaker/speaker's bureau fees from Janssen, Gilead, Roche, AbbVie, Novartis, Celgene, Adaptive Biotechnologies, BioGene, and AstraZeneca; research support/research funding from Janssen, Gilead, Roche, AbbVie, BeiGene, and AstraZeneca; travel, accommodations, expenses from Janssen, Roche, Novartis, AbbVie, Gilead, and Celgene. MF received honoraria from Janssen and Gilead. JAG-M received honoraria for advisory board and speaker's bureau from Mundipharma, Glaxo, AbbVie, Roche, Gilead, AstraZeneca, and Janssen; research support from Hoffman-La Roche, AbbVie, and Janssen. RG-S received educational grants from AbbVie, Janssen, and Novartis. EG received travel grants, honoraria as a consultant and/or speaker bureau for Janssen-Cilag, Roche, and AbbVie. MGDS received honoraria for consultancy/advisory boards with Roche, Janssen Cilag, Gilead, AbbVie, and BMS; research Grant from Gilead. YH received honoraria from AbbVie, Janssen, AstraZeneca, and Roche, outside the submitted work. JAH-R received honoraria and advisory boards fees from Janssen, AbbVie, AstraZeneca, Roche, Beigene, Gilead, and BMS-Celgene. OJ received honoraria from AbbVie, Janssen, and Roche. APK received honoraria from Janssen, BMS, AstraZeneca, Roche/Genentech; research money from Janssen, BMS, AstraZeneca, and Roche/Genentech. SK received Travel grant from Celgene; received research funding from Janssen and AbbVie. LL received honoraria from Roche, AbbVie, Janssen, and AstraZeneca. M-DL received travel expenses and advisory board compensation from Janssen, AbbVie, and Roche. AL-G received speaker's bureau fees from Roche, Janssen, AbbVie, Celgene, Fresenius, Novonordisk; received advisory board participation fees from Janssen, and AbbVie; received travel and accommodation expenses from Roche, Janssen, and AbbVie. MMar received speaker bureau (invited speech) fees from Amgen; received honoraria as a consultant from Gilead. JM received honoraria and travel grants from AbbVie, Janssen, Roche, Gilead, and Takeda. MMat received research grant from GILEAD. FRM received research funding from Gilead; received advisory board participation fees from AbbVie, Gilead, Janssen, AstraZeneca, Takeda, and Roche; received speakers bureau fees from Gilead, Janssen, and AbbVie. TM received honoraria from AbbVie, Janssen, AstraZeneca, Gilead, Roche, and Alexion; received advisory board participation fees from AbbVie, AstraZeneca, Janssen, Alexion, Morphosys, and Sunesis. RM received honoraria from Janssen, and AbbVie. CUN received research funding and/or consultancy fees from AbbVie, AstraZeneca, Janssen, CSL Behring, and Takeda. MAP received advisory board participation fees from Janssen, AbbVie, AstraZeneca, and Merck; received speaker's bureau fees from Janssen, AbbVie, AstraZeneca, Varifarma, and Merck. FMQ received advisory board participation fees from AstraZeneca and Janssen; received speakers bureau fees from Janssen. GR received consultancy fees and honoraria from AbbVie, AstraZeneca, and Janssen. GMR received honoraria from AbbVie, Gilead, and Janssen; received research funding from Gilead. MŠi received consultancy fees, advisory board participation fees, travel grants, and honoraria from Janssen, Gilead, Roche, AstraZeneca, and AbbVie. MŠp received 
honoraria from AbbVie, AstraZeneca, Gilead, Janssen, and Roche. PS received funding from Gilead; received advisory board participation fees from AbbVie and Janssen; received honorarium AbbVie, Janssen, and AstraZeneca. LT received advisory board participation fees from Janssen, Roche, AbbVie, Gilead, Takeda; research funding from Janssen, Roche, Takeda, and Gilead. EVDS participated in teaching activities for Amgen. MV received advisory board participation fees from Janssen, Roche, AstraZeneca; received travel expenses from Janssen and AbbVie. AV received speaker's bureau fees from Italfarmaco and Gilead; received advisory board participation fees from Janssen and Takeda. CV received honoraria from Janssen. TW received research funding from Roche; received honoraria for advisory board, and research funding from Janssen; received honoraria, advisory board participation fees, and travel grant from AbbVie; received speaker's bureau fees from Gilead. LYSS received advisory board participation fees from Gilead-Kite, Janssen, AbbVie, AstraZeneca, Beigene, Roche, Pfizer, Jazz, BMS, and Merck; received speaker's bureau fees from Janssen, AbbVie, AstraZeneca, Gilead-Kite, Roche, Pfizer, and Merck. MC received honoraria from AbbVie, Gilead, Janssen, and AstraZeneca. AC received speaker's bureau and advisory board fees from AbbVie, AstraZeneca, Gilead, and Janssen. RF received speaker's bureau fees and/or advisory board participation fees from Amgen, Incyte, Janssen, Pfizer, AbbVie, Novartis, Servier, AstraZeneca, ACu received speaker's bureau fees and advisory board participation fees from Abbvie, Asta-Zeneca, Gilead, Janssen KS received honoraria and research support from Janssen, Abbvie, AstraZeneca, Gilead. PG received honoraria from AbbVie, Arqule/ MSD, AstraZeneca, Celgene/Juno/BMS, Janssen, Loxo/Lilly, Roche; Research support, AbbVie, AstraZeneca, Janssen, Gilead, Sunesis. TC, AK, GK, AAC, MB, DB, ACa, SC, J-GC, CC-G, LDP, MRDP, GDP, CD, MDi, DD, ME, SE-A, AE, BEs, LF, AF, HF, MFü, MG, AG, OG, YKH, TH, II, GI, AJ, OBK, EK, LKK, JL, DL, EL, LM, RM, IM, FM, MMor, MMot, RNR, JO, LO, $M P, I P, V M P, G Q, K Q, R R, G S, A S, O S, N S, T$, DTR, SHT, MVG, RVK, EWS, MY, AR, EM have no conflict of interest to disclose.

\section{ADDITIONAL INFORMATION}

Supplementary information The online version contains supplementary material available at https://doi.org/10.1038/s41375-021-01450-8.

Correspondence and requests for materials should be addressed to Kostas Stamatopoulos or Paolo Ghia.

Reprints and permission information is available at http://www.nature.com/ reprints

Publisher's note Springer Nature remains neutral with regard to jurisdictional claims in published maps and institutional affiliations.

\begin{abstract}
Open Access This article is licensed under a Creative Commons Attribution 4.0 International License, which permits use, sharing, adaptation, distribution and reproduction in any medium or format, as long as you give appropriate credit to the original author(s) and the source, provide a link to the Creative Commons license, and indicate if changes were made. The images or other third party material in this article are included in the article's Creative Commons license, unless indicated otherwise in a credit line to the material. If material is not included in the article's Creative Commons license and your intended use is not permitted by statutory regulation or exceeds the permitted use, you will need to obtain permission directly from the copyright holder. To view a copy of this license, visit http://creativecommons. org/licenses/by/4.0/.
\end{abstract}

(c) The Author(s) 2021

${ }^{1}$ Hematology Department and HCT Unit, G. Papanicolaou Hospital, Thessaloniki, Greece. ${ }^{2}$ Institute of Applied Biosciences, Centre for Research and Technology Hellas, Thessaloniki, Greece. ${ }^{3}$ Università Vita-Salute San Raffaele and IRCC Ospedale San Raffaele, Milan, Italy. ${ }^{4}$ Department of Mathematics, University of loannina, loannina, Greece. ${ }^{5}$ Centre for Atherothrombosis and Metabolic Disease, Hull York Medical School, Hull, UK. ${ }^{6}$ Spanish Society of Haematology and hemotherapy (SEHH: Sociedad Española de Hematología y hemoterapia), Madrid, Spain. ${ }^{7}$ Hematology Department, Hospital Universitario de La Princesa, Madrid, Spain. ${ }^{8}$ Department of Hematology and Central Hematology Laboratory, Inselspital, Bern University Hospital, University of Bern, Bern, Switzerland. ${ }^{9}$ University Clinical Center of Serbia, Belgrade, Serbia. ${ }^{10}$ School of Medicine, University of Belgrade, Belgrade, Serbia. ${ }^{11}$ Hospital Clinico Universitario de Salamanca (CAUSA / IBSAL), Salamanca, Spain. ${ }^{12}$ Department of Immunology, Genetics and Pathology, Science for Life Laboratory, Uppsala University, Uppsala, Sweden. ${ }^{13}$ Department of Clinical Genetics, Uppsala University Hospital, Uppsala, Sweden. ${ }^{14}$ Inst J Bordet (ULB), Brussels, Belgium. ${ }^{15}$ IRCSS Ospedale San Raffaele, Milan, Italy. ${ }^{16}$ Hematology Unit, 1st Dept of Internal Medicine, AUTH, AHEPA Hospital, Thessaloniki, Greece. ${ }^{17}$ Department of Hematology, Health Research Institute IIS-FJD, Fundacion Jimenez Diaz University Hospital, Madrid, Spain. ${ }^{18}$ Hospital Clínic of Barcelona, Barcelona, Spain. ${ }^{19}$ Hematology Unit Terrassa Hospital, Terrasa, Spain. ${ }^{20}$ Division of Hematology, Department of Translational Medicine, Università del Piemonte Orientale Amedeo Avogadro, Azienda Ospedaliero-Universitaria Maggiore della Carità Novara, Novara, Italy. ${ }^{21}$ UOC Ematologia PO Vito Fazzi Lecce, Lecce, Italy. ${ }^{22}$ Department of Biomedicine and Prevention Hematology, University Tor Vergata, Rome, Italy. ${ }^{23} 1$ st Internal Medicine Department, Propaedeutic, Hematology Clinical Trial Unit, National and Kapodistrian University of Athens, Athens, Greece. ${ }^{24}$ Belfast City Hospital, Belfast, Northern Ireland. ${ }^{25}$ Department of Internal Medicine - Hematology and Oncology, University Hospital, Brno; Department of Medical Genetics and Genomics, Faculty of Medicine, Masaryk University, Brno, Czech Republic. ${ }^{26}$ Department of Haematology Athens Medical CenterPsychikon Branch, Athens, Greece. ${ }^{27}$ Department I of Internal Medicine, Center for Integrated Oncology Aachen Bonn Cologne Duesseldorf (CIO ABCD), University Hospital Cologne, University of Cologne, Cologne, Germany. ${ }^{28}$ Clinical Hematology Unit, Oncology Center, Faculty of Medicine, Mansoura University, Mansoura 35516, Egypt. ${ }^{29}$ Hospital Italiano La Plata, Buenos Aires, Argentina. ${ }^{30}$ Pathology Service, Hospital del Mar, Barcelona, Spain. ${ }^{31}$ Hematology, Fondazione IRCCS Istituto Nazionale Tumori, Milan, Italy. ${ }^{32}$ Hematology Unit, Azienda Unità Sanitaria Locale - IRCCS, Reggio Emilia, Italy. ${ }^{33}$ Division of Hematology, AO S. Croce e Carle, Cuneo, Italy. ${ }^{34}$ Department of Hematology, Odense University Hospital, Odense, Denmark. ${ }^{35}$ Hematology Department, Hospital Universitario Puerta de Hierro-Majadahonda, Madrid, Spain. ${ }^{36}$ Department of Hematology, Hospital General Universitario, Valencia, Spain. ${ }^{37}$ Fundación de Investigación del Hospital General Universitario, Valencia, Spain. ${ }^{38} \mathrm{Hematology}$ Unit AO Cosenza, Cosenza, Italy. ${ }^{39}$ Department of Hematology, Hospital del Mar, Barcelona, Spain. ${ }^{40}$ Department of Hematology, Rigshospitalet, Copenhagen University Hospital, Copenhagen, Denmark. ${ }^{41}$ Hematology Department, Portuguese Institute of Oncology, Lisbon, Portugal. ${ }^{42}$ Department of Hematology, Shamir Medical Center, Zerifin, Israel. ${ }^{43}$ Sackler Faculty of Medicine, Tel Aviv University, Tel Aviv, Israel. ${ }^{44}$ Hematology Center after Prof. Yeolyan MH RA, Yerevan, Armenia. ${ }^{45}$ Department of Hematology, Tel Aviv Sourasky Medical Center and Sackler School of Medicine, Tel Aviv University, Tel Aviv, Israel. ${ }^{46}$ Hematology Department Infanta Leonor University Hospital, Madrid, Spain. ${ }^{47}$ Laboratory for Leukemia Diagnostics, Department of Medicine III, University Hospital, LMU Munich, Munich, Germany. ${ }^{48}$ Dipartimento di Diagnostica per Immagini, Radioterapia Oncologica ed Ematologia, Fondazione Policlinico Universitario Agostino Gemelli IRCCS, Rome, Italy. ${ }^{49}$ Rabin Medical Center, Petah Tikva, and the Sackler School of Medicine, Tel-Aviv University, Tel-Aviv, Israel. ${ }^{50}$ Department of Hematology, University hospital Dubrava, Zagreb, Croatia. ${ }^{51}$ Department of Hematology, Universitaire ziekenhuizen Leuven, Herestraat 49, 3000 Leuven, Belgium. ${ }^{52}$ Federal State Budgetary Educational Institution of Higher Education Academician I.P. Pavlov First St. Petersburg State Medical University of the Ministry of Healthcare of Russian Federation, St. Petersburg, Russia. ${ }^{53}$ Department and Clinic of Hematology, Blood Neoplasms and Bone Marrow Transplantation Wroclaw Medical University, Pasteura Street 4, 50-367 Wroclaw, Poland. ${ }^{54}$ Dept of Hematology, Cancer Center Amsterdam, Amsterdam University Medical Centers, University of Amsterdam, Amsterdam, the Netherlands. ${ }^{55}$ Department of Hematology, Haga Teaching Hospital, The Hague, The Netherlands. ${ }^{56} \mathrm{Hematology}$ Department, Unit Research, Complejo Asistencial Universitario de Burgos, Burgos, Spain. ${ }^{57}$ Department of Internal Medicine, Postgraduate Institute of Medical Education and Research, Chandigarh, India. ${ }^{58}$ Sezione di Ematologia, Dipartimento di Scienze Radiologiche ed Ematologiche, Università Cattolica del Sacro Cuore, Rome, Italy. ${ }^{59}$ Department of Internal Medicine, Albert Schweitzer hospital, Dordrecht, the Netherlands. ${ }^{60}$ Department of Hematology, Santa Chiara Hospital, Trento, Italy. ${ }^{61}$ Hematology and Stem Cell Transplant Center Marche Nord Hospital, Pesaro, Italy. ${ }^{62}$ Dept of Medical Sciences, Section of Hematology, University of Modena and Reggio E., Modena, Italy. ${ }^{63} \mathrm{Hematology}$ Unit and BM Transplant Center, AO SS Antonio e Biagio e Cesare Arrigo, Alessandria, Italy. ${ }^{64} \mathrm{Hematology}$ Department, Ramón y Cajal University Hospital, Madrid, Spain. ${ }^{65}$ Department of Hematology, Uppsala University Hospital, Uppsala, Sweden. ${ }^{66}$ Hematology, Department of Translational and Precision Medicine, Sapienza University, Rome, Italy. ${ }^{67}$ Faculty of Medicine, Clinical Centre of Vojvodina, University of Novi Sad, Novi Sad, Serbia. ${ }^{68}$ Hematology Department Hospital Universitario 12 de Octubre, Madrid, Spain. ${ }^{69}$ Experimental Hematooncology Department, Medical University of Lublin, Lublin, Poland. ${ }^{70}$ Hematology Department, St. John's Cancer Center, Lublin, Poland. ${ }^{71}$ S.C. Ematologia ASST Spedali Civili Brescia, Brescia, Italy. ${ }^{72}$ Consultant Haematologist, St James's Hospital, Leeds LS9 7TF, UK. ${ }^{73}$ Hematology and Stem Cell Transplantation Unit, Ospedale Oncologico A. Businco, ARNAS "G. Brotzu", Cagliari, Italy. ${ }^{74} \mathrm{Hematology}$ Clinic, ASUFC, Udine, Italy. ${ }^{75}$ S.C. Ematologia, Città della Salute e della Scienza di Torino, Turin, Italy. ${ }^{76}$ FUNDALEU, Clinical research center Buenos Aires, Buenos Aires, Argentina. ${ }^{77}$ Consultative Hematology Department with a Day 


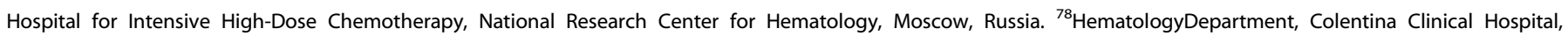

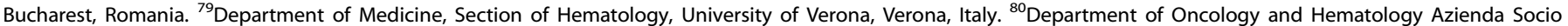

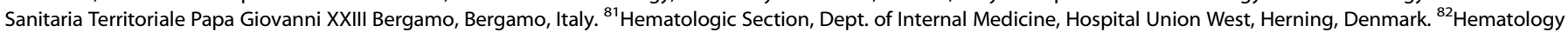
Unit, Foundation IRCCS Ca' Granda Ospedale Maggiore Policlinico of Milan, Milan, Italy. ${ }^{83}$ St. Anna University Hospital, Ferrara, Italy. ${ }^{84}$ Department of Hematology, Shaare-

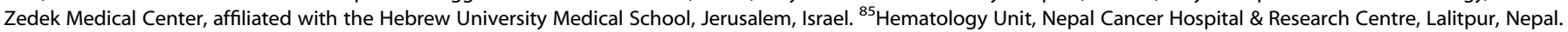

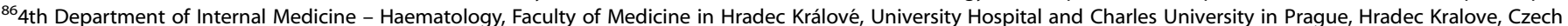

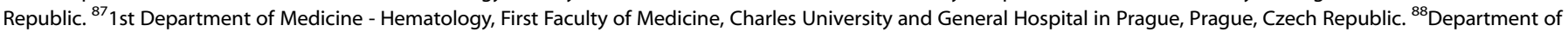

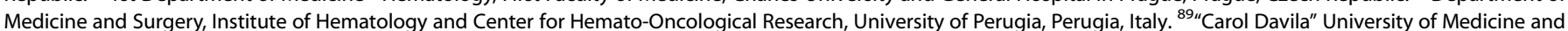

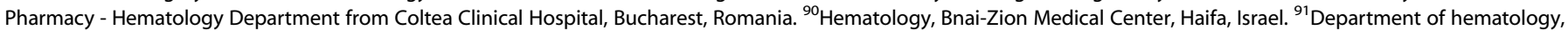

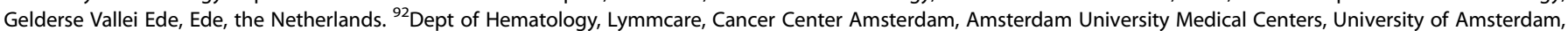

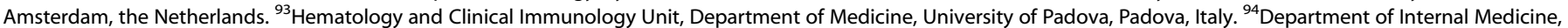

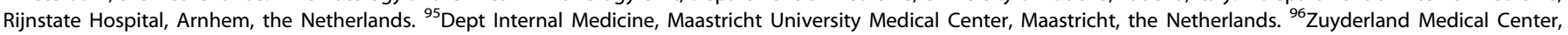

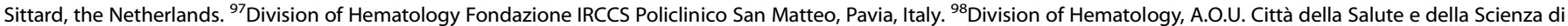





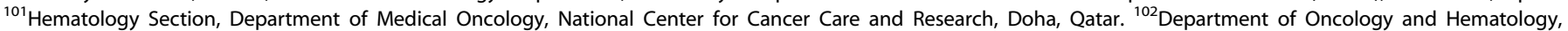

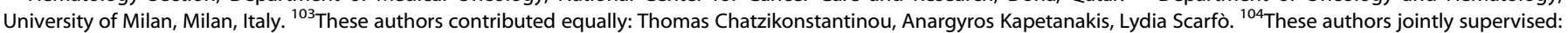
Kostas Stamatopoulos, Paolo Ghia.『email: kostas.stamatopoulos@certh.gr; ghia.paolo@hsr.it 